\title{
Subject index to volume 3 (1999)
}

Adaline

Aggregation rules

Artificial data generation

Artificial neural networks

Association rules

Batches

Change-point

Characterization

Classification

Classification trees

Classifier comparison

Classifier selection

Clustering

Combining classifiers

Concept drift

Concept learning

Concept learning algorithm evaluation

Constraint satisfaction

Constructive induction

Constructive learning algorithms

Consumer product data

Correlated distribution matrix (CDM)

Data analysis

Data mining

Dataset morphology

Decision lists

Decision table

Decision trees

Demographic variables

Dependency relationships

Dimensionality optimization

Discrimination

Dynamic probabilistic networks

EEG

Empirical testing

Estimation theory

Feature selection

Function approximation

Fuzzy controller
Fuzzy graphs 37

Fuzzy learning 139

Fuzzy PID controller 127

Fuzzy set 363

Genetic algorithms 211

Goal programming $\quad 75$

Graph match 413

Heuristic learning 211

Historical data 399

Incremental learning 453

Inductive learning 227, 399

Inference break-up method 127

Information flow 287

Interpretation $\quad 37$

Knowledge discovery 211, 413

Latent semantic indexing (LSI) $\quad 377$

Learning 37

Learning from examples $\quad 95$

Least absolute deviations $\quad 75$

Machine learning $\quad 1,245,491$

Magnetic suspension system 127

Maintenance rules $\quad 475$

Marketing and tourism 491

Markov processes $\quad 287$

Mathematical programming $\quad 245$

Membership function $\quad 139$

Merging order 139

Method of potential functions $\quad 307$

Molecular biology 413

Multidimensional metrics $\quad 319$

Multinomial logistic regression 23

Multiple models 191

Multivariate decision trees

Multivariate statistics $\quad 491$

Nearest neighbor $\quad 191$

Neural networks $\quad 55,307$

Noise reduction $\quad 267$

1088-467X/99/\$ - see front matter (c) 1999 Elsevier Science B.V. All rights reserved.

PII: S $1088-467 \times(99) 00037-2$ 
Parallel database server

Parallel processing

Pattern classification

Perceptron

Piecewise regression

Planning

Projection

Purchase behaviors

Quantitative value

Reasoning with different levels of abstraction

Reconfiguration of software modules

Robust regression

Rule extraction
Sample C4.5 211

Science data analysis $\quad 159$

Self-organizing map $\quad 111$

Simulated annealing $\quad 177$

Statistical hypothesis testing $\quad 287$

Structural databases 413

Transaction 363

Tumor classification $\quad 287$

Vector quantization $\quad 111$

Visualization $\quad 111$

Voting 191

Wavelet transform $\quad 267$

Wide-area surveillance 339 